\title{
ROLE OF ULTRASOUND IN RETINOPATHY OF PREMATURITY: A CASE REPORT
}

\author{
Khaladkar SM, Kotkar R, Kulkarni A, Joshi A
}

Deenanath Mangeshkar Hospital and research centre, Maharashtra, India

DOI: http://doi.org/10.4038/sljr.v3i1.43

Keywords: Retinopathy of prematurity, retrolental fibroplasia, leukokoria, prematurity, high oxygen therapy

\section{Introduction}

Retinopathy of prematurity (ROP) is a cause of leucokoria. Other causes of white pupil are congenital cataract, retinoblastoma and pseudoglioma ${ }^{1}$.

ROP is also known as Terry syndrome and was previously known as retrolental fibroplasia (RLF). It is an ocular disorder affecting prematurely born babies who have received oxygen therapy as intensive neonatal care for underdeveloped lungs. It is caused by disorganized growth of retinal blood vessels which subsequently leads to scarring and retinal detachment. Both hypoxia and oxygen toxicity contribute to the development of $\mathrm{ROP}^{2}$.

\section{Case Report}

A 7 month old female infant was referred for orbital ultra sonography (USG) with a history of bilateral diminution of vision. She did not make eye contact and there was leukokoria on examination. There was a history of premature delivery at 30 weeks with oxygen therapy. USG of both orbits was performed with high resolution linear probe (7-12 MHz). Axial length of both eye balls was $10 \mathrm{~mm}$. Approximately $11 \mathrm{~mm}$ x 5 $\mathrm{mm}$ well-defined echogenic lesion was noted in the anterior vitreous in the retrolental region in both eye balls containing a few cystic components with no calcification or vascularity. No retinal detachment was seen in either eye ball (Fig 1, 2). There was no evidence of primitive hyperplastic persistent vitreous (PHPV). A diagnosis of retinopathy of prematurity Stage 3 was made. The patient underwent lensectomy, vitrectomy and membranectomy on both sides.

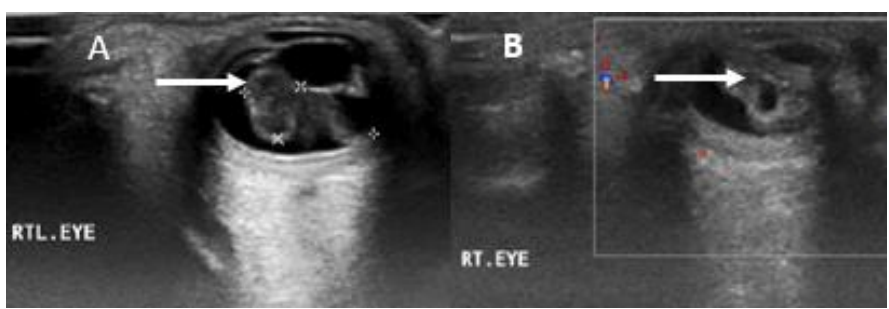

Figure 1. (A)B mode ultrasound of right orbit demonstrating lesion (marked by white arrow) with both solid and cystic components in retrolental region. (B) Lesion with no vascularity on colour Doppler. There is no evidence of retinal detachment.

Corresponding Author: Khaladkar SM <drsanjaymkhaladkar@gmail.com>

https://orcid.org/0000-0001-6415-5368

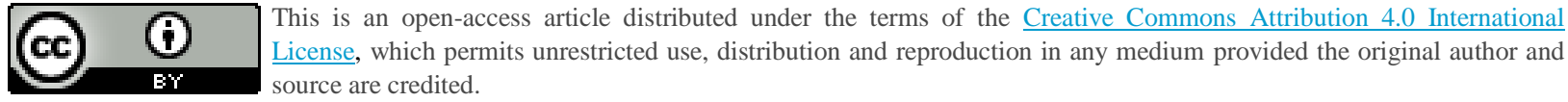




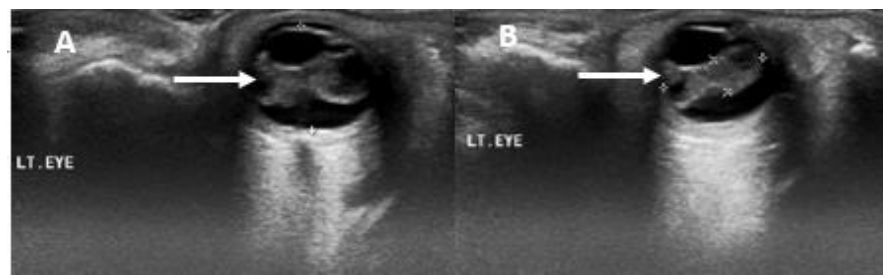

Figure 2.(A and B)B mode ultrasound of left orbit demonstrating lesion (marked by white arrows) with both solid and cystic components in retrolental region with no evidence of retinal detachment.

\section{Discussion}

Low birth weight, low gestational age and prolonged exposure to supplementary oxygen therapy following delivery are associated with $\mathrm{ROP}^{3}$. ROP develops in $16 \%$ of all premature births, $60 \%$ of infants with birth weight of less than $1500 \mathrm{~g}$ and $65 \%$ if the birth weight is less than $1250 \mathrm{~g}$. Respiratory distress and intraventricular hemorrhage are predictive of the development of ROP. ROP occurs in extremely low birth weight infants in high income countries, with declining incidence in last few decades. Due to high rates of premature birth and increasing resuscitation of premature infants with suboptimal standards of care, a third epidemic of ROP has ensued in middle income countries ${ }^{3}$. Its incidence can decrease with better understanding of screening and management of these patients ${ }^{4}$.

Foetal retina begins to develop vascularization by $13^{\text {th }}$ to $17^{\text {th }}$ weeks of pregnancy. ROP develops if the blood vessels grow and branch abnormally with development of fibrous tissue along the new vessels which is called fibrovascular proliferation. Retinal detachment occurs on contraction of fibrous tissue ${ }^{2}$.
Patients with ROP are at a greater risk of developing refractive errors, cataract, glaucoma, strabismus, amblyopia, myopia, retinal detachment and blindness in later life. Hence, early detection of ROP is a must to prevent, diagnose and treat these conditions $^{5}$. All infant with gestational age of 31 weeks or less regardless of birth weight, birth weight of $1250 \mathrm{~g}$ or less and premature babies with severe illnesses in the perinatal period should be screened for $\mathrm{ROP}^{6}$.

Stages of ROP on fundoscopy are based on findings at the junction between the vascularised and avascular retina. Stage 1: a faint demarcation line, Stage 2: an elevated ridge, Stage 3: extra retinal fibrovascular tissue, Stage 4: subtotal retinal detachment and Stage 5: total retinal detachment ${ }^{6}$.

On USG, ROP is bilateral and often asymmetric. Eye balls are normal in size. There is formation of retrolental medium level echogenic mass with occasional calcification in anterior vitreous in stage 3 disease with clear space between the mass and retina or empty vitreal space. Shrinkage of ocular axis is not a feature. Retinal detachment is seen in stage 4 and 5 disease. USG is extremely useful in differentiating between stage 3 and stage 4 or 5 disease. Hence it should be incorporated in screening programmes of at risk premature babies to avoid development of complications like blindness $^{6,7,8}$.

USG in our case referred for bilateral leucokoria showed bilateral retrolental echogenic lesions with cystic components and clear space between the lesion and retina with no calcification or vascularity. 
Above features ruled out PHPV and retinoblastoma. There was no evidence of retinal detachment on either side. These features helped in diagnosing stage three disease of ROP which was useful in management and prognostification. Bilateral lensectomy, vitrectomy and membranectomy was offered.

Treatment options are peripheral retinal ablation by laser photocoagulation, cryotherapy, intra-vitreal injection of avastin, vitrectomy for stage 4 and 5 ROP and scleral buckling. Oral propranolol has been shown to reduce progression of $\mathrm{ROP}^{9}$.

Differential diagnoses include persistent hyperplastic primary vitreous (PHPV) which is typically unilateral and occurs in term babies and familial exudative vitreo retinopathy a genetic disorder that disrupts retinal vascularization in full term infants ${ }^{8}$.

\section{Take home message:}

This case supports the literature on the need of screening premature and low birth weight babies having prolonged exposure to supplementary oxygen therapy following delivery to avoid development of future complications suchas blindness, using $\mathrm{B}$ scan USG as it can detect retinal detachment with ROP.

\section{References.}

1. Patel N, Salchow DJ. Materin M.Differentials and approach to
leukocoria.Conn Med. 2013;77(3):13340.PMID: 23589950

2. Good WV. Retinopathy of prematurity and the peripheral retina. J Pediatr. 2008 Nov;153(5):591-2.PMID: 18940346 PMCID: PMC2603064

3. Zin A, Gole GA. Retinopathy of Prematurity - Incidence today. Clin Perinatol 2013 Jun; 40(2):185- 200.PMID: 23719304.

4. Hakeem AH, Mohamed GB, Othman MF.Retinopathy of prematurity: a study of prevalence and risk factors.Middle East Afr J Ophthalmol. 2012 Jul-Sep;19(3):289-94 PMID: 22837621 PMCID: PMC3401797.

5. Chen J, Smith LE .Retinopathy of prematurity. Angiogenesis. 2007;10(2):13340. PMID: 17332988.

6. Jefferies AL. Canadian Paediatric Society, Fetus and Newborn Committee.Retinopathy of prematurity: An update on screening and management. Paediatr Child Health.2016 Mar;21(2):101-8,PMID: 27095887 PMCID: PMC4807789.

7. Afshari M, Hart L, Afshari N, Mukai S. Ophthalmic ultrasonography in children. Int Ophthalmol Clin. 2001;41:153-164.

8. Aironi VD, Gandage SG. Pictorial essay: Bscan ultrasonography in ocular abnormalities.Indian J Radiol Imaging. 2009 Apr-Jun;19(2):109-15. PMID: 19881064 PMCID: PMC2765186.

9. Salvin JH, Lehman SS, Jin J, Hendricks DH.Update on retinopathy of prematurity: treatment options and outcomes. Curr Opin Ophthalmol. 2010 Sep;21(5):329-34.PMID: 20634698 